\title{
SHARPENING AND POSITIONING OF REGIONS OF INTEREST IN ATOM PROBE SAMPLES USING IN-SITU SPUTTERING
}

\author{
D. J. Larson*, A. Cerezo**, Y. Q. Ma**, T. J. Godfrey**, P. H. Clifton* and G. D. W. Smith** \\ * Recording Head Operations, Seagate Technology, Bloomington, MN 55435, USA \\ ** Department of Materials, Oxford University, Oxford, UK OX13PH
}

Successful atom probe analysis [1] of a material requires a needle-shaped specimen that has 1) the appropriate radius of curvature and 2) the desired region of interest (ROI) present within a few nanometers of the sample apex. In the case of analysis of samples fabricated from planar thin films [2], where the ROI may be only a few tens of nanometers, this task is particularly challenging, as shown in Fig. 1. A sample fabricated to have the ROI present near the apex, but with a large radius of curvature $(>\sim 100 \mathrm{~nm})$, or one that has the proper radius $(\sim 50 \mathrm{~nm})$ but the ROI positioned too far from the apex, is not acceptable. Previously, inert gas ion sputtering in situ during field emission (FE) imaging has been used to sharpen atom probe samples or to study the effects of ion sputtering on a metal surface [3-5]. Sputtering is accomplished by applying a negative potential to a field ion (FI) sample to induce field emission in the presence of $\mathrm{Ne}$ gas atoms (pressure $\sim 10^{-4} \mathrm{mbar}$ ). Emitted electrons ionize the $\mathrm{Ne}$ gas atoms and the electric field from the sample accelerates the ions back to the sample, from which they remove material by ion sputtering $[3,4]$. This process may be used not only to sharpen samples, but also position the specimen apex at the ROI. The current work presents a method by which atom probe samples are modified by this technique to have the correct geometry and be accurately positioned for thin film analysis. Although the current example shows how this technique is applied to thin films, it is equally applicable to any sample with a specific ROI.

To employ this process, a sample is prepared from a thin film geometry using a previously described method employing focused ion beam milling [2]. Fig. 2 shows a series FI and FE images that generally illustrate the current method, as applied to a sample consisting of a silicon substrate onto which is deposited the thin films of interest, together with a protective cap layer. The sample is field evaporated in FI mode to whatever voltage is required to produce a full, uniform image, Fig. 2a. The sample polarity is reversed and a voltage applied to produce a low FE current, Fig. 2b. During FE sputtering, the initial crystallographic similarities evident between the FI and FE images disappear due to sputter damage. For most sample geometries, the radius will decrease slightly during sputtering [3] and the applied voltage is lowered in order to maintain a constant current and sputter rate. After a short sputtering time, the sample appears as shown in Fig. 2c. FI imaging at this stage, Fig. 2d, produces a relatively full image at a voltage several $\mathrm{kV}$ below that shown in Fig. 2a, and reveals an ion-damaged surface. Field evaporation to remove the damaged region yields the image shown in Fig. 2e, which is very similar to that shown in Fig. 2 a but is obtained at a lower voltage. This process has resulted in a sample whose apex position has been moved down toward the ROI shown in Fig. 1 and also has been slightly sharpened. Repeated application of this process produces a significantly sharpened sample (radius reduced by a factor of $\sim 1.5$ ) with a darkly-imaging $5 \mathrm{~nm} \mathrm{Cu}$ layer near the apex in FI imaging mode (dark annulus shown in Fig. 2f). The geometry of a sample that has been subjected to a similar procedure, and is ready for atom probe analysis, is shown in Fig. 3 [6].

1. M. K. Miller et al., Atom Probe Field Ion Microscopy (Oxford University Press, Oxford, 1996).

2. D. J. Larson et al., Microscopy and Microanalysis 7, 24 (2001).

3. P. Janssen and J. P. Jones, J. Phys. D 4 (1971) 118

4. R. Smith, J. Phys. D 17 (1984) 1045

5. G. L. Kellogg, J. Vac. Sci. Technol. A 2, 1597 (1984).

6. We are grateful to the EPSRC for financial support under grant number GR/N09060/01 


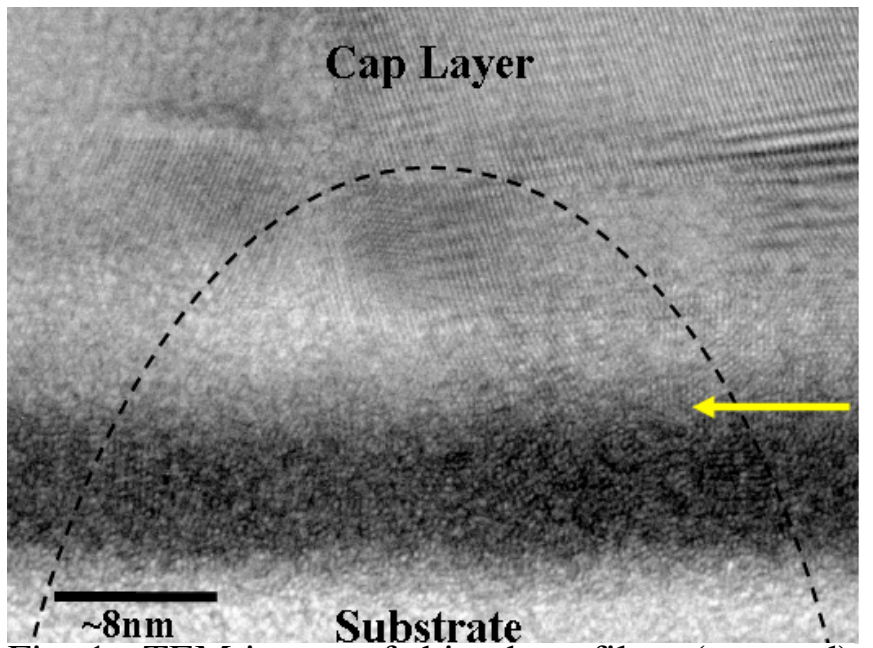

Fig. 1. TEM image of thin sheet films (arrowed) and protective capping layer deposited onto a silicon substrate. The dashed line shows the approximate shape required in order to fabricate an atom probe specimen from this structure.

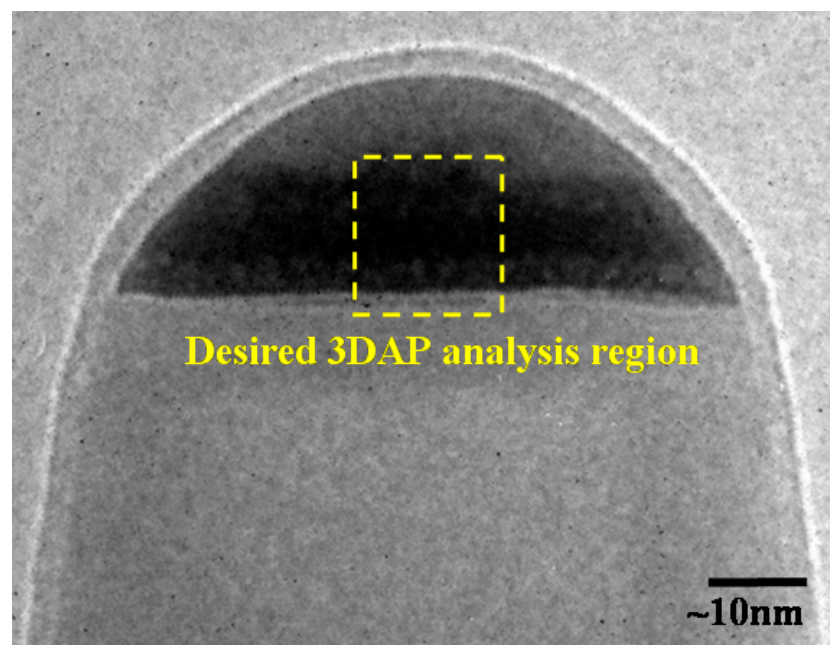

Fig. 3. TEM image of an atom probe sample after FIB fabrication [2], in-situ sputtering and FI imaging. A portion of the protective cap layer is visible above the darkly imaging films in the region of interest.

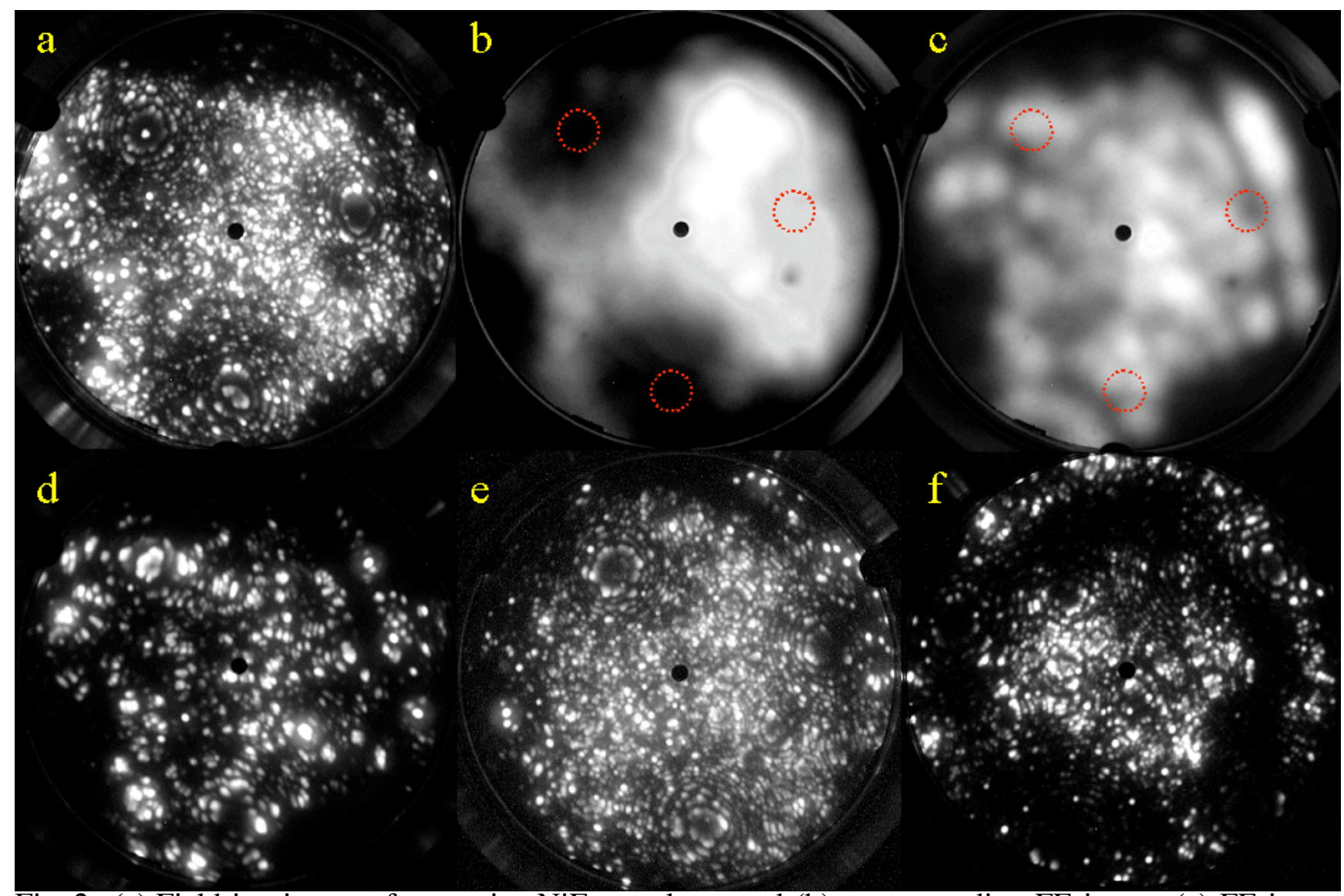

Fig. 2. (a) Field ion image of protective NiFe cap layer and (b) corresponding FE image, (c) FE image after sputtering using a low FE current, (d) FI image after sputtering showing damaged surface region, (e) FI image (still in NiFe cap layer) at after evaporation of damaged region and (f) final FI image after several repeats of this sputtering procedure. A darkly imaging $5 \mathrm{~nm} \mathrm{Cu}$ layer is now visible as a dark annulus just outside the brightly-imaging $\mathrm{NiFe}$ region in the center. 